\title{
THE SPACE OF $\omega$-LIMIT SETS OF A CONTINUOUS MAP OF THE INTERVAL
}

\author{
ALEXANDER BLOKH, A. M. BRUCKNER, P. D. HUMKE, AND J. SMÍTAL
}

\begin{abstract}
We first give a geometric characterization of $\omega$-limit sets. We then use this characterization to prove that the family of $\omega$-limit sets of a continuous interval map is closed with respect to the Hausdorff metric. Finally, we apply this latter result to other dynamical systems.
\end{abstract}

\section{INTRODUCTION AND PRELIMINARIES}

Let $I$ be a closed interval in $\mathbb{R}$ and let $\mathcal{C}(I, I)$ denote the class of continuous functions mapping $I$ into $I$. For $f \in \mathcal{C}(I, I)$, let $f^{0}$ be the identity function, and for $n \in \mathbb{N}, n \geq 0$, let $f^{n+1}=f \circ f^{n}$. The functions $f^{n}$ are called the iterates of $f$. A set $W \subset I$ is called an $\omega$-limit set for $f$ if there exists an $x \in I$ such that $W$ is the cluster set of the sequence $\left\{f^{n}(x)\right\}$. We denote this set by $\omega_{f}(x)$ or $\omega(x)$, and we denote the class of all $\omega$-limit sets for $f$ by $\omega_{f}$ or $\omega$. If $W \in \omega_{f}$, then $W$ is a closed nonempty subset of $I$ and $f(W)=W$. When furnished with the Hausdorff metric, $\omega_{f}$ becomes a subspace of the compact metric space $\mathcal{K}$ of all closed nonempty subsets of $I$. The space $\omega_{f}$ played a central role in [3] where forms of chaos in terms of the continuity structure of the map $x \rightarrow \omega_{f}(x)$ were studied. Related questions were also considered in [1] and [4].

In this paper we prove that $\omega_{f}$ is a closed subspace of $\mathcal{K}$, and is therefore compact. This is somewhat surprising since the map $x \rightarrow \omega_{f}(x)$ is far from continuous. We do this in Section 3, where we also obtain some other ways in which $\omega$-limit sets combine to produce other $\omega$-limit sets. Finally, in Section 4 we show that our main results carry over to certain other dynamical systems.

The proof that $\omega_{f}$ is closed in $\mathcal{K}$ depends on an extension of a well-known property of $\omega$-limit sets. This property states that in order for $W$ to be in $\omega_{f}$, it is necessary that for each nonempty proper closed subset $F$ of $W$, the set $\overline{f(W \backslash F)}$ intersects $F$. Loosely speaking, this property implies that $f$ and its iterates create a certain amount of "transportation" of some points in a relatively open subset

Received by the editors January 3, 1995.

1991 Mathematics Subject Classification. Primary 26A18, 54H20,58F03,58F08.

Key words and phrases. Interval maps, Hausdorff metric, $\omega$-limit sets, periodic points.

This work was partially done during the first author's visit to MSRI (the research at MSRI funded in part by NSF grant DMS-9022140) whose support the first author acknowledges with gratitude. The fourth author (J. S.) was supported in part by the Grant Agency of Czech Republic, Grant No. 201/94/1088. Much of the work was accomplished during separate visits by authors 1,3 , and 4 to Santa Barbara; these authors wish to express their gratitude to the Department of Mathematics at the University of California-Santa Barbara and specifically to Andy Bruckner for his kind hospitality. 
$G=W \backslash F$ of $W$ to points outside of $G$. Actually, of course, much more transportation is necessary - the iterates must be able to transport points in $G$ "all over" $W$. We make this statement precise in Section 2. There we obtain (in Theorem 2.12) a characterization of $\omega$-limit sets in terms of a transportation condition on the sets. This characterization provides a convenient method of verifying that sets we consider are indeed $\omega$-limit sets.

Although the following pull back lemma is well known, we use it liberally throughout the paper and include a statement for completeness.

Lemma 1.1. Let $I$ be a closed interval, $f: I \rightarrow I$ be continuous surjective and suppose $J \subset I$ is an interval [a closed interval]. Then there is an interval [a closed interval $L \subset I$ such that $f(L)=J$ and no point of int $L$ maps to an endpoint of $J$.

In some sense, $L$ is an "exact preimage" of $J$.

\section{A CRiterion For a SET to BE AN $\omega$-Limit SET}

We begin this section with a simple example. Let $h$ be the well-known hat function:

$$
h(x)= \begin{cases}2 x, & 0 \leq x<1 / 2 \\ 2(1-x), & 1 / 2 \leq x \leq 1\end{cases}
$$

It is easy to verify that the set

$$
H_{1}=\{0\} \cup \bigcup_{n=0}^{\infty}\left\{\frac{1}{2^{n}}\right\}
$$

is an $\omega$-limit set for $h$. If we extend $h$ to $\bar{h}$ on $[-1,1]$ by

$$
\bar{h}(x)= \begin{cases}h(x), & 0 \leq x<1 \\ -h(x), & -1 \leq x \leq 0,\end{cases}
$$

then both $H_{1}$ and

$$
H_{2}=\{0\} \cup \bigcup_{n=0}^{\infty}\left\{\left(-\frac{1}{2^{n}}\right)\right\}
$$

are $\omega$-limit sets, but $H_{1} \cup H_{2}$ isn't. This example shows that two $\omega$-limit sets can intersect without their union being an $\omega$-limit set. Observe that while every neighborhood of the common point 0 expands under the iterates of $\bar{h}$ to all of $[-1,1]$, unilateral neighborhoods don't. There is no transportation from points near $H_{1} \backslash\{0\}$ to points near $H_{2} \backslash\{0\}$.

In this section we study carefully certain questions involving the expansion of unilateral neighborhoods under the iterates. A set $V$ is a right (left) unilateral neighborhood of $x$ if there is an $\epsilon>0$ such that $[x, x+\epsilon) \subset V((x-\epsilon, x] \subset V)$; also if $T$ is a side of $x$ (i.e. $T$ means either "left" or "right") then we can talk about $T$-unilateral neighborhoods of $x$. Our main result, Theorem 2.12, shows that if every point of a nonempty closed set $A$ has a unilateral neighborhood that properly expands under the iterates, then $A$ is an $\omega$-limit set. In particular, our example would not have worked if $H_{1}$ and $H_{2}$ had 0 as a limit point from the same side.

We continue with the following construction. Let $\mathbf{i}=\left\{I_{1}, I_{2}, \ldots\right\}$ be a sequence of (not necessarily disjoint) intervals. Despite the fact that many of the 
THE SPACE OF $\omega$-LIMIT SETS OF A CONTINUOUS MAP OF THE INTERVAL 1359

objects we are about to introduce depend on the sequence $\mathbf{i}$, to simplify notation we will suppress specific reference to the sequence $\mathbf{i}$ whenever possible. Let $B^{N}=\bigcup_{j=1}^{N} I_{j}, 1 \leq N<\infty$, and $B=\bigcup_{j=1}^{\infty} I_{j}$. Then, $B^{N}$ has finitely many components, $J_{1}^{N}, \ldots, J_{k_{N}}^{N}$ and for any point $x \in B$ if $N$ is sufficiently large, the component $J^{N}(x)$ of $B^{N}$ containing $x$ is well defined and non-empty. For such $N$ we have $J^{N+1}(x) \supset J^{N}(x)$. Let $J(x)=\bigcup_{i=1}^{\infty} J^{i}(x)$. Then $J(x)$ is an interval of some kind (open, semi-open or closed), and the definition implies that two sets $J(x)$ and $J(y)$ are either disjoint or equal. We call the sets $J(x)$ the pieces of the sequence $\mathbf{i}$. It's important to note that a piece of the sequence $\mathbf{i}$ is contained in a connected component of $B$ but does not necessarily coincide with that component. For example, if $I_{1}=[0,1 / 2]$ and $I_{n}=[1 / 2+1 /(n+1), 1 / 2+1 / n]$ for $n \geq 2$ then $B=\bigcup_{j=1}^{\infty} I_{j}=[0,1]$, but there are two pieces of this sequence of intervals, $[0,1 / 2]$ and $(1 / 2,1]$. In short, the set $B$ alone (i.e. union of all intervals) does not determine the pieces of the underlying sequence.

Lemma 2.1. Let $\mathbf{i}=\left\{I_{1}, I_{2}, \ldots\right\}$ be a sequence of intervals and suppose $J(x)$ contains a T-unilateral neighborhood of $x$. Then there exists $n$ such that $I_{n}$ contains a $T$-unilateral neighborhood of $x$.

Proof. As $J(x)$ contains a $T$-unilateral neighborhood of $x$, there is an $r$ such that $J^{r}(x)$ contains a $T$-unilateral neighborhood of $x$. Since every $J^{r}(x)$ is a finite union of intervals from $\mathbf{i}$ the result follows.

The next lemmas provide us with the tools used in subsequent investigations. To state them we need some additional definitions. An interval $J$ is called wandering if $f^{m}(J) \cap f^{n}(J)=\emptyset$ whenever $m>n \geq 0$. Let $U=U_{1} \cup \cdots \cup U_{N}$ be a union of pairwise disjoint non-degenerate closed intervals. For any set $K \subset U$ let $f_{U}(K)=$ $f(K) \cap U$ (this may be empty; for example, $f_{U}(K)=f(K) \cap U=\emptyset$ for any set $K$ if $f(U)$ is disjoint from $U)$. Inductively define $f_{U}^{2}(K)=f_{U}\left(f_{U}(K)\right)=f_{U}(f(K) \cap U)=$ $f(f(K) \cap U) \cap U$ etc. In other words, $f_{U}^{n}(K)$ is the image of the set of those points in $K$ which remain in $U$ under the first $n$ iterates of $f$. Let $\widetilde{K} \equiv \widetilde{K}(U)=\bigcup_{i=1}^{\infty} f_{U}^{i}(K)$; although $\widetilde{K}$ depends on $U$, to avoid convoluted notation we use $\widetilde{K}$ whenever the set $U$ is evident.

Lemma 2.2. Let $K$ be an interval [closed interval] and $n$ be a nonnegative integer. Then there exists a pairwise disjoint set of intervals [closed intervals], $\left\{L_{s}^{n}: 1 \leq\right.$ $\left.s \leq i_{n}\right\} \subset K$ such that

$$
f_{U}^{n}(K)=I_{1}^{n} \cup I_{2}^{n} \cup \cdots \cup I_{i_{n}}^{n}
$$

where $f^{n}\left(L_{s}^{n}\right)=I_{s}^{n}$ and $\bigcup_{i=1}^{n} f^{i}\left(L_{s}^{n}\right) \subset U$ for every $1 \leq s \leq i_{n}$. Moreover, if

$$
\mathbf{k} \equiv \mathbf{k}(K, U)=\left\{I_{s}^{n}: 1 \leq s \leq s_{n} ; n=1,2, \ldots\right\}
$$

and a piece of the sequence $\mathbf{k}$ contains a T-unilateral neighborhood of $x \in \widetilde{K}$ then there are integers $n$ and $1 \leq s \leq i_{n}$ and an interval $W \subset L_{s}^{n}$ such that $f^{n}(W)$ is a $T$-unilateral neighborhood of $x$.

Proof. The lemma is clearly true for $n=0$. Suppose it holds for $n$. That is, suppose

$$
f_{U}^{n}(K)=I_{1}^{n} \cup I_{2}^{n} \cup \cdots \cup I_{i_{n}}^{n}
$$

where $\left\{L_{s}^{n}: 1 \leq s \leq i_{n}\right\} \subset K$ are pairwise disjoint intervals, $f^{n}\left(L_{s}^{n}\right)=I_{s}^{n}$ and $\bigcup_{i=1}^{n} f^{i}\left(L_{s}^{n}\right) \subset \bar{U}, 1 \leq s \leq i_{n}$. For each $1 \leq s \leq i_{n}, f\left(I_{s}^{n}\right)$ is an interval and so $f\left(I_{s}^{n}\right) \cap U$ is the union of at most $N$ disjoint intervals. The collection of all of these 
intervals forms the set, $\left\{I_{s}^{n+1}: 1 \leq s \leq i_{n+1}\right\}$. Let $I_{s}^{n+1}$ be one such interval. Then $I_{s}^{n+1}$ has an exact $f$-preimage interval in some $I_{t}^{m}$ and this $f$-preimage interval has an exact $f^{n}$-preimage [closed] interval in $L_{t}^{n} \subset K$; this last interval is then $L_{s}^{n+1}$. For fixed $s$, the intervals, $I_{t}^{n+1}$ comprising $f\left(I_{s}^{n}\right) \cap U$ are disjoint and so the corresponding $L_{t}^{n+1} \subset L_{s}^{n}$ are disjoint. The fact that $\left\{L_{t}^{n+1}\right\}$ are pairwise disjoint now follows from the induction assumption that $\left\{L_{s}^{n}\right\}$ are pairwise disjoint. Note also that if $K$ is closed then the $L_{s}^{n}$ and $I_{s}^{n}$ intervals can be chosen closed as well. Finally the second part of the lemma follows from the definitions, Lemma 2.1 and Lemma 1.1.

For us, the iterative construction used to verify Lemma 2.2 is at least as important as the lemma itself and we make explicit use of it in the sequel. The pieces of $\mathbf{k}$ will be the subject of a good deal of our attention. We first note that the set of pieces of $\mathbf{k}$ is quasi-invariant under $f$ in the sense that if $J$ is any piece of $\mathbf{k}$, then each component of $f_{U}(J)$ is contained in a single piece of $\mathbf{k}$.

Lemma 2.3. Let $K \subset U$ be an interval. Then the following hold.

(1) $\widetilde{K}$ is the union of two disjoint sets: a finite union, $\mathcal{A}$, of disjoint intervals and a finite union, $\mathcal{B}$, of pairwise disjoint orbits of wandering intervals. Moreover, all the aforementioned intervals are pieces of $\mathbf{k} ;$ also, if $K$ is closed then so are all of the wandering intervals in question.

(2) Let $H$ be one of the intervals described in property (1), and suppose $H$ contains a T-unilateral neighborhood of $x$. Then there is an interval $W \subset K$ such that $f^{n}(W)$ is a non-degenerate $T$-unilateral neighborhood of $x$ and $f(W) \cup f^{2}(W) \cup$ $\cdots \cup f^{n}(W) \subset U$

Proof. First notice that property (2) follows from (1) and second part of Lemma 2.2. We now turn to the proof of (1). If $f^{j}(K) \cap U=\emptyset$ for some $j$, the statement of the lemma is trivially true since in this case $\widetilde{K}$ is a finite union of intervals. So we may assume that $f^{j}(K) \cap U \neq \emptyset$ for every $j$. It follows that either the entire orbit of $K$ is contained in $U$ or there is a minimal $n_{o}$ such that $f^{n_{o}}(K) \not \subset U$ and in this latter case we may assume $f^{n_{o}}(K)$ contains an endpoint of $U$.

Case 1. The entire orbit of $K$ is contained in $U$.

If $K$ is wandering we immediately get the required representation for $\widetilde{K}$. Suppose, then, that the orbit of $K$ intersects itself; more precisely, suppose $f^{n}(K) \cap$ $f^{n+m}(K) \neq \emptyset$. Then all the sets $T_{j}=\bigcup_{i=0}^{\infty} f^{n+j+m i}(K), 0 \leq j \leq m-1$ and $f^{s}(J), 0<s<n$, are intervals so by construction each is contained in a single piece of $\mathbf{k}$. Thus, these finitely many pieces of $\mathbf{k}$ give the required representation of $\widetilde{K}$;

Case 2. There is a minimal $n_{o}$ such that $f^{n_{o}}(K) \not \subset U$ and $f^{n_{o}}(K)$ contains an endpoint of $U$.

For $i<n_{o}, f^{i}(K)$ is a subinterval of $U$ and we include those pieces of $\mathbf{k}$ which contain one of these subintervals into $\mathcal{A}$. Also include into $\mathcal{A}$ those pieces, $\left\{J_{i}: i=\right.$ $1,2, \ldots, l\}$, of $\mathbf{k}$ which contain endpoints of $U$; we refer to such pieces as endpieces of $\mathbf{k}$. We begin with $\mathcal{B}=\emptyset$. Considering the endpieces, $J$, in order, we add them to $\mathcal{A}$ and then elements of their orbits to $\mathcal{A}$ or $\mathcal{B}$ according to the following hierarchical rules:

1. Suppose $f^{m}(J) \subset U$ is disjoint from $\mathcal{A} \cup \mathcal{B}$ for $1 \leq m<n$ and $f^{n}(J) \cap X \neq \emptyset$ for some $X \in \mathcal{A} \cup \mathcal{B}$. If $n>1$ we add to $\mathcal{A}$ those pieces of $\mathbf{k}$ which contain 
intervals $f^{m}(J), 1 \leq m<n$. Note that in this case, every component of $f^{n}(J) \cap U$ is contained in a piece of $\mathbf{k}$ which is in $\mathcal{A}$ or a piece which is in $\mathcal{B}$

2. Suppose $f^{m}(J) \subset U$ is disjoint from $\mathcal{A} \cup \mathcal{B}$ for $m<n$ and $f^{n}(J) \cap U=\emptyset$. Again, for $n>1$ we add to $\mathcal{A}$ those pieces of $\mathbf{k}$ which contain intervals $f^{m}(J)$, $m<n$.

Note that if $f^{n}(J) \cap U \neq \emptyset$ and $f^{n}(J) \cap U^{c} \neq \emptyset$ then $f^{n}(J)$ intersects an endpiece of $\mathbf{k}$.

3. Suppose $f^{n}(J) \subset U$ for every $n \in \mathbb{N}$ and that $f^{n}(J) \cap X=\emptyset$ for every $X \in \mathcal{A} \cup \mathcal{B}$. Let $I_{k}$ be a piece of $\mathbf{k}$ containing $f^{k}(J)$. Then because these are pieces, $f\left(I_{k}\right) \subset I_{k+1}$. If the sequence $\left\{I_{1}, I_{2}, \ldots\right\}$ is eventually periodic, we add all its finitely many intervals to $\mathcal{A}$. Otherwise we claim that for some $M$ and any $k>M$ we must have $f\left(I_{k}\right)=I_{k+1}$. Indeed, there are only finitely many endpieces; suppose that $M$ is sufficiently large that for each endpiece whose orbit intersects $\bigcup_{i=1}^{\infty} I_{i}$ there exists an integer $i<M$ such that some image of the endpiece in question maps into $I_{i}$. Also suppose that for some $k>M$ we have $f\left(I_{k}\right) \subset I_{k+1}$, where $I_{k} \neq I_{k+1}$. It follows that for some $y \in K$ and integer $r$ we have that $f^{j}(y) \in U, 0 \leq j \leq r-1, f^{r}(y) \in I_{k+1} \backslash f\left(I_{k}\right)$. Let $L \ni y$ be the largest interval such that $f^{j}(L) \subset U, 0 \leq j \leq r$. Then the maximality of $L$ implies that either it coincides with $K$ (the previous Case 1) or for some $j \leq r, f^{j}(L)$ is contained in an endpiece (in this case $f^{r}(y) \in I_{k+1} \backslash f\left(I_{k}\right)$ is impossible due to the choice of $M$ and the fact that the intervals $I_{1}, I_{2}, \ldots$ are pairwise disjoint). So indeed for $k>M$ we have $f\left(I_{k}\right)=I_{k+1}$. Now, we add all $I_{k}, 1 \leq k \leq M$, to $\mathcal{A}$ and $I_{k}, M<k$, to $\mathcal{B}$.

This completes the process of adding pieces of $\mathbf{k}$ to $\mathcal{A}$ and orbits consisiting of pieces to $\mathcal{B}$. Note that by the construction, $\mathcal{B}$ consists of finitely many orbits of wandering intervals and all intervals in all these orbits are pieces of $\mathbf{k}$.

Let $x \in \widetilde{K}$. Then there is a least integer $n$ such that $x \in f_{U}^{n}(K)$. If $n<n_{o}$, then $x \in f^{n}(K) \subset U$ and the entire piece of $\mathbf{k}$ which contains $f^{n}(K)$ was put into $\mathcal{A}$. If $n=n_{o}$, then $x$ is in an endpiece so again was introduced into $\mathcal{A}$. Suppose that $n>n_{o}$. Then $x$ is in the union of pieces which contain the orbit of an endpiece; by the construction it follows that $x$ is either in an interval from $\mathcal{A}$ or in an interval from $\mathcal{B}$. This completes the first part of the proof.

It remains to show that if $K$ is closed and $J^{*}$ is a wandering piece, then $J^{*}$ is also closed. To this end we need the following construction. Let $R \supset J^{*}$ be the component of $U$ containing $J^{*}$, and denote the family of all endpieces and the interval $f(K)$ by $\mathcal{V}$ where $f(K)$ is included in the family $\mathcal{V}$ only if $F(K) \subset U$. Let $I \neq J^{*}, I \in \mathcal{V}$. Then either the entire $f$-orbit of $I$ is contained in $U$ or there is a minimal $\ell \equiv \ell(I)$ such that $f^{\ell}(I) \not \subset U$. In the first case there are two possibilities. It may happen that for some $\ell \equiv \ell(I)$ we have $f^{\ell}(I) \subset J^{*}$; then set $J^{\prime}(I)=I$. Otherwise the orbit of $I$ is disjoint from $J^{*}$ and we set $J^{\prime}(I)=\emptyset$. Consider the second case. If for some $k<\ell$ the interval $f^{k}(I)$ is not disjoint from $J^{*}$ then due to quasi-invariance we have in fact $f^{k}(I) \subset J^{*}$; in that case set $J^{\prime}(I)=I$. Finally let $I, f(I), \ldots, f^{\ell-1}(I)$ be all disjoint from $J^{*}$. Clearly $f^{\ell}(I)$ is an interval which intersects each component of $U$ over another interval. If $f^{\ell}(I)$ is disjoint from $J^{*}$ we set $J^{\prime}(I)=\emptyset$. Otherwise due to quasi-invariance $f^{\ell}(I) \cap R=M$ is a subinterval of $J^{*}$. By Lemma 1.1 there is an interval $L \subset I$ such that $f^{\ell}(L)=M$ and if $M$ is closed then we may assume that so is $L$; otherwise neither $M$ nor $L$ is closed. 
Set $L=J^{\prime}(I)$ and denote by $\mathcal{D}$ the finite family of those $I \in \mathcal{V}$ for which $J^{\prime}(I)$ is non-empty.

For each $I \in \mathcal{D}$ there is a number $\ell(I)$ such that $f^{\ell(I)}\left(J^{\prime}(I)\right)=f^{\ell(I)}(I) \cap J^{*}$. We show that $\bigcup_{I \in \mathcal{D}} f^{\ell(I)}\left(J^{\prime}(I)\right)=J^{*}$. If $y \in J^{*}$ then there is a point $z \in K$ and a unique $n \in \mathbb{N}$ such that $f^{n}(z)=y$ and $\bigcup_{i=0}^{n-1} f^{i}(z) \subset U$. The fact that $n$ is unique follows from the fact that $J^{*}$ is wandering. Consider the maximal number $m<n$ such that $f^{m}(z)$ is contained in an interval $Z \in \mathcal{V} ; m$ is well defined since $z \in K$. Let us show that $Z \in \mathcal{D}$ and $n-m=\ell(Z)$. Indeed, none of the points $f^{m+1}(z), \ldots, f^{n-1}(z)$ is contained in an interval from $\mathcal{V}$. This implies that $f(Z) \subset U, \ldots, f^{n-m-1}(Z) \subset U$ and so the result follows from the construction.

Assume that contrary to what we want to prove, the interval $J^{*}$ is not closed. Then for at least one interval $I \subset \mathcal{D}$ we have that neither $f^{\ell(I)}\left(J^{\prime}(I)\right) \cap J^{*}$ nor $J^{\prime}(I)$ is closed either. It is easy to see then that $J^{\prime}(I)$ is located at one of the endpoints of a non-closed interval $I \in \mathcal{D}$. Indeed, otherwise $\overline{J^{\prime}(I)} \subset I$; since $U$ is closed we see that $f^{i}\left(\overline{J^{\prime}(I)}\right) \subset U, 0 \leq i \leq \ell(I)$, which due to the definitions implies that $f^{\ell(I)}\left(\overline{J^{\prime}(I)}\right) \subset J^{*}$ contradicting the assumption that $f^{\ell(I)}\left(J^{\prime}(I)\right) \cap J^{*}$ is not closed. We now repeat the construction replacing $J^{*}$ in it by the non-closed interval $J^{\prime}(I)$ defined above. This is possible since the fact that $J^{\prime}(I)$ is wandering follows from the fact that $J^{*}$ is wandering. However after finitely many steps we will inevitably get to the point when the current non-closed interval is located at the same endpoint of a non-closed interval $I \in \mathcal{D}$ at which one of its predecessors was found. Since all the non-closed intervals in the construction map one into another under appropriate powers of $f$ we see that the interval in question is not wandering after all. This contradiction completes the proof.

We defined the sets $\mathcal{A}$ and $\mathcal{B}$ of Lemma 2.3 in order that the proof be as direct as possible. It's easy to see, however, that the statement itself is robust enough to allow us to transfer the initial portion of any orbit from the set $\mathcal{B}$ to the set $\mathcal{A}$ without changing the statement of the lemma. In order to make future proofs as notationally simple as we can, in the sequel, we will always include the pieces of $\mathcal{B}$ which contain components of $f_{U}(K)$ into $\mathcal{A}$.

The goal of this section is to find geometric conditions which characterize $\omega$-limit sets. This study culminates in Theorem 2.12 which shows that for interval maps there is a convenient geometric condition on a set $A$ which is equivalent to the fact that $A$ is an $\omega$-limit set. First we need a few preliminary facts.

Let $A \subset[0,1]$ be a closed set and $x \in A$. We say that a side $T$ (either "right" or "left") of a point $x$ is $A$-covering if for any union of finitely many closed intervals $U$ such that $A \subset$ int $U$ and any closed $T$-unilateral neighborhood $V(x)$ there are finitely many components of $\widetilde{V}(x)$ such that the closure of their union covers $A$. For clarity, we have chosen to couch the previous definition in a traditional setting. There are other options, however, which more closely fit the dynamics we are describing as we will see in Lemma 2.4 where we show we can define $A$-covering side replacing the word "components" by the word "pieces" in the definition. It follows immediately from the definition and Lemma 2.3 that if $T$ is an $A$-covering side of $x$ then no point of $A$ is in a wandering component of $\widetilde{V}(x)$. Indeed, otherwise there would be infinitely many closed components of $\widetilde{V}(x)$ covering $A$ contradicting the definition of an $A$-covering side. 
If $T$ is an $A$-covering side of $x$ then any $T$-unilateral neighborhood $V(x)$ is also said to be A-covering.

Lemma 2.4. Let $A \subset[0,1]$ be a closed set and $x \in A$. Then $T$ is an A-covering side of $x$ if and only if for any union of finitely many closed intervals $U$ such that $A \subset$ int $U$ and any $T$-unilateral neighborhood $V(x)$ there are finitely many pieces of $\mathbf{k}(V(x), U)$ such that the closure of their union covers $A$.

Proof. Fix $U$. By definition, each piece of $\mathbf{k}(V(x), U)$ is contained in a component of $\widetilde{V}(x)$. So if $V(x)$ is a $T$-unilateral neighborhood with the property that there are finitely many pieces of $\mathbf{k}(V(x), U)$ such that the closure of their union covers $A$, then the closure of the union of the components of $\widetilde{V}(x)$ containing these pieces will also contain $A$. Hence, the property involving pieces of $\mathbf{k}(V(x), U)$ implies that the side $T$ of $x$ is an $A$-covering side. Suppose that $T$ is an $A$-covering side of $x$. By Lemma 2.3 the family of pieces of $\mathbf{k}\left(V_{T}(x), U\right)$ consists of finitely many pieces forming the subfamily $\mathcal{A}$ and some closed wandering pieces. The fact that wandering pieces are closed implies that they are in fact components of $\widetilde{V}_{T}(x)$, so adding those of them which are included in the family of components covering $A$ to $\mathcal{A}$ we get a finite family of pieces of $\mathbf{k}(V(x), U)$ such that the closure of their union covers $A$.

If every $x \in A$ has an $A$-covering side we call the set $A$ locally expanding. Relying upon Lemma 2.4 we use Lemmas 2.1 and 2.3 to study locally expanding sets. We begin with a series of simple but useful lemmas.

Although the property of invariance is not an explicit part of the definition of locally expanding, it is easy to see that it is implicit.

Lemma 2.5. A locally expanding set $A$ is invariant.

Proof. Let $A$ be locally expanding and suppose that $x \in A$ but $f(x) \notin A$. Then there exists a union of finitely many intervals $U \supset A$ such that for any sufficiently small neighborhood $V$ of $x$ we have $f(V) \cap U=\emptyset$. The definition of $\widetilde{V}$ implies that $\widetilde{V}=\emptyset$ which is a contradiction.

Note that $f_{U}(K)$ rather than $K$ is used as the starting point in defining $\widetilde{K}$ specifically to exclude singletons which are not $f$-fixed points from the collection of locally expanding sets.

We now turn to the case when a locally expanding set $A$ has a non-empty interior.

Lemma 2.6. If $A$ is locally expanding with non-empty interior, then $A$ is a cycle of intervals and $f \mid A$ is transitive.

Proof. Let $J$ be an interval contained in $A$. If $J$ is wandering, then the $f$-orbit of $J$ is disjoint from $J$. Hence, for any union of closed intervals $U$ such that int $U \supset A$ it follows that $\widetilde{J} \cap J=\emptyset$ contradicting the fact that $A$ is locally expanding. Using this and the fact that $A$ is invariant, it is easy to find a component, $J$, of $A$ with non-empty interior such that for some $n \in \mathbb{N}, J, f(J), \ldots, f^{n-1}(J)$ are disjoint and $f^{n}(J) \subset J$.

If there is a point $x \in A \backslash \bigcup_{i=0}^{n-1} f^{i}(J)$, then the distance between $x$ and $\bigcup_{i=0}^{n-1} f^{i}(J)$ is positive and so the closure of the orbit of $J$ does not contain $x$. This contradicts the fact that $A$ is locally expanding at interior points of $J$.

Hence, $A=\bigcup_{i=0}^{n-1} f^{i}(J)$ and as any open subset of $A$ has a dense orbit in $A, f \mid A$ is transitive. 
Due to Lemma 2.6 we will often divide our proofs into two parts corresponding to the nowhere dense case and to the case of a cycle of intervals.

Lemma 2.7. If $A$ is locally expanding or an $\omega$-limit set then $f(A)=A$.

Proof. The case of an $\omega$-limit set is trivial and well known. Let $A$ be a locally expanding set. If $A$ is a cycle of intervals the statement is clear. Let $A$ be nowhere dense and suppose $f(A) \subsetneq A$. Using the continuity of $f$ it is easy to find a finite union of intervals $U=U_{1} \cup \cdots \cup U_{n}$ with the following properties:

1. $\emptyset \neq U_{1} \cap A \subset A \backslash f(A)$.

2. $U_{k}, U_{k+1}, \ldots, U_{n}$ are the components of $U$ which contain points from $f(A)$.

3. For any $x \in U$ if $f(x) \in U$ then $f(x) \in U_{k} \cup U_{k+1} \cup \cdots \cup U_{n}$.

Let $W=U_{k} \cup U_{k+1} \cup \cdots \cup U_{n}, x \in A$, and let $V$ be a neighborhood of $x$. Then, the definition of $U$ implies that any $\widetilde{V} \subset W$. But $A$ is not contained in the closure of $\widetilde{V}$ since $\widetilde{V} \cap U_{1}=\emptyset$. This contradicts the hypothesis that $A$ is locally expanding and hence $f(A)=A$ as claimed.

The following property of $\omega$-limit sets is well known.

Lemma 2.8 (Sharkovsky [5]; cf. also [2, Lemma IV.3]). Let $W$ be an $\omega$-limit set of $f$ and let $F$ be a closed subset of $W$, with $W \backslash F \neq \emptyset$. Then the closure of $f(W \backslash F)$ intersects $F$.

Lemma 2.9 establishes this same property for locally expanding sets.

Lemma 2.9. Let $A$ be a locally expanding set and let $F \neq A$ be a closed subset of $A$. Then the closure of $f(A \backslash F)$ intersects $F$.

Proof. Suppose to the contrary that $\overline{f(A \backslash F)} \cap F=\emptyset$. It follows from Lemmas 2.6 and 2.8 that $A$ is not a cycle of intervals and hence $A$ must be nowhere dense. Let $\varepsilon$ denote the distance between the sets $F$ and $\overline{f(A \backslash F)}$ and $\delta<\varepsilon / 2$ be such that if $|z-\zeta|<\delta$ then $|f(z)-f(\zeta)|<\varepsilon / 2$. As $A$ is nowhere dense, $A$ can be covered by a finite pairwise disjoint collection of intervals, $\left\{U_{i}: i=1,2, \ldots, U_{n}\right\}$ each of whose lengths is less than $\delta$. The choice of $\delta$ entails that the union of those intervals $U_{i}$ disjoint from $F$ is nonempty; we let $W$ denote this union. If $U_{i} \subset W$ then $f\left(U_{i}\right)$ is an interval shorter than $\varepsilon / 2$ which intersects $f(A \backslash F)$ and so is disjoint from $F$. That is, if $x \in W$ and $f(x) \in U$ then $f(x) \in W$ which contradicts the assumption that $A$ is locally expanding at such $x$.

Corollary 2.10. Let $A$ be an $\omega$-limit set or a locally expanding set. Then:

- A does not contain an isolated periodic point unless it is finite and if $A$ is finite then it is a periodic orbit.

- if $A$ is infinite and $x$ is an isolated point of $A$, then there is a sequence of distinct points $x_{0}=x, x_{-1}, \ldots$ and open intervals $I_{0} \ni x_{0}, I_{-1} \ni x_{-1}, \ldots$ such that $f\left(x_{-n-1}\right)=x_{-n}$ and $f^{n}\left(I_{-n} \cap A\right)=\left\{x_{0}\right\}$; in particular the set of all preimages of $x$ in $A$ is infinite.

Proof. To prove the first property, we let $y \in A$ be an isolated periodic point of period $n$ and set $B=\bigcup_{i=0}^{n-1} f^{-i}(y) \cap A$. Then $B$ an invariant closed set. On the other hand if $U$ is a neighborhood of $y$ sufficiently small for $U \cap A=\{y\}$ then $B=\bigcup_{i=0}^{n-1} f^{-i}(U) \cap A$ and so $B$ is open in the relative topology. However by Lemma 2.8 (in case $A$ is an $\omega$-limit set) or by Lemma 2.9 (in case $A$ is a locally expanding set) there are no invariant proper subsets of $A$ which are both open and 
THE SPACE OF $\omega$-LIMIT SETS OF A CONTINUOUS MAP OF THE INTERVAL 1365

closed. Therefore $B=A$. By Lemma 2.7, $f(A)=A$, so $A=f^{n}(A)=\bigcup_{i=0}^{n-1} f^{i}(y)$ is a periodic orbit.

The second property follows immediately from the first and the fact that $f$ is continuous and $f \mid A$ is onto.

The following proposition is a technical tool to be used in the proof that locallyexpanding sets are $\omega$-limit sets.

Proposition 2.11. Let $A$ be a locally expanding set and $U=U_{1} \cup \cdots \cup U_{n}$ be a union of closed intervals such that $A \subset$ int $U$ and $U_{i} \cap A \neq \emptyset$ for every $i$. Let $z \in U_{1}$ and $V$ be an A-covering closed unilateral neighborhood of $z$. Then there is a point $\zeta \in U_{n}$, a closed interval $V^{\prime} \subset V$ and an $m \in \mathbb{N}$ such that

- $\bigcup_{i=1}^{m} f^{i}\left(V^{\prime}\right) \subset U$,

- every component of $U$ contains at least one of these intervals, and

- $f^{m}\left(V^{\prime}\right)$ is an A-covering unilateral neighborhood of $\zeta$.

Proof. We first prove the proposition when $n=2$ as this case is easily generalized to arbitrary $n \in \mathbb{N}$.

Consider $\widetilde{V}$. By Lemma 2.3, $\widetilde{V}$ is the union of two sets: $\mathcal{A}=D_{1} \cup \cdots \cup D_{s}$ where each $D_{i}$ is a piece of $\mathbf{k}(V, U)$ and $\mathcal{B}$, the union of finitely many orbits of closed wandering intervals which are also pieces of $\mathbf{k}(V, U)$. Moreover, all these intervals are pairwise disjoint. As we have seen before, since $A$ is locally expanding, $A \subset \overline{\mathcal{A}}$. CASE 1. $A \cap U_{2}$ is infinite.

There is at least one interval $D_{i}$ such that for some point $y$ we have $y \in \operatorname{int} U_{2} \cap$ int $D_{i} \cap A$. As $A$ is locally expanding, $y$ has an $A$-covering side and so by part (2) of Lemma 2.3 there is an interval $V^{\prime} \subset V$ and a number $\ell$ such that $\bigcup_{i=1}^{\ell} f^{i}\left(V^{\prime}\right) \subset U$ and $f^{\ell}\left(V^{\prime}\right)$ is an $A$-covering unilateral neighborhood of $y$.

CASE 2. $A \cap U_{2}$ is finite.

Let $y \in$ int $U_{2} \cap A$. Since $y$ is isolated in $A$ we can apply Corollary 2.10 to find infinitely many preimages of $y$ in $A$. Hence, there is an interval $V^{\prime} \subset V$ and a number $\ell$ such that $\bigcup_{i=1}^{\ell} f^{i}\left(V^{\prime}\right) \subset U$ and $f^{\ell}\left(V^{\prime}\right)=Z^{\prime}$ is an $A$-covering unilateral neighborhood of a point $y^{\prime}$ which is an $f^{p}$-preimage of $y$. Moreover, it also follows from Corollary 2.10 that $y$ is not periodic so that the points $f^{i}\left(y^{\prime}\right), i \geq 0$, are distinct.

Our aim is to show that $f^{p}\left(Z^{\prime}\right)$ contains an $A$-covering unilateral neighborhood of $y$. Indeed, if this is the case, the conclusion then follows from Lemma 1.1. If in fact $f^{p}\left(Z^{\prime}\right)$ contains a neighborhood of $y$ then since $y$ has an $A$-covering side, $f^{p}\left(Z^{\prime}\right)$ contains an $A$-covering unilateral neighborhood of $y$. Suppose that $f^{p}\left(Z^{\prime}\right)$ is a $T$-unilateral neighborhood of $y$ and let us show that $T$ is an $A$-covering side of $y$. It is enough to show that for any $T$-unilateral neighborhood $R$ of $y$ the set $\widetilde{R}$ has finitely many components such that $A$ is contained in the closure of their union. Let $S$ be a unilateral neighborhood of $y^{\prime}, S \subset Z^{\prime}$ so that $f^{p}(S)=D \subset R$ is a $T$-unilateral neighborhood of $y$ and $\bigcup_{i=1}^{p-1} f^{i}(S) \subset U$. Moreover, by Corollary 2.10 we may assume that $f^{i}\left(f^{p-i}(S) \cap A\right)=\{y\}$ for any $0 \leq i \leq p$; in particular $f^{p}(S) \cap A=D \cap A=\{y\}$. Also, since all the points $y^{\prime}, f\left(y^{\prime}\right), \ldots, f^{p}\left(y^{\prime}\right)=y, f^{p+1}\left(y^{\prime}\right)$ are distinct we can choose $S$ sufficiently small that the sets $S, f(S), \ldots, f^{p+1}(S)$ are pairwise disjoint. 
ClaIm: If $u \in f^{i}(S) \cap A, 1 \leq i \leq p$, then $u$ has infinitely many preimages in $A$ lying outside $C=\bigcup_{i=1}^{p} f^{i}(S)$.

Clearly it is enough to show that every point $u \in f^{i}(S)$ has at least one preimage outside $C=\bigcup_{i=1}^{p} f^{i}(S)$. We prove the claim by applying this statement whenever the next preimage of $u$ happens to lie in $C$ and making use of the fact that $u$ is not periodic. If there is a point in $f^{-1}(u) \cap A$ which is not contained in $C$ then this is the preimage of $u$ we are looking for. If $f^{-1}(u) \cap A \subset C$, then because of the choice of $S$ we have $f^{-1}(u) \cap A \subset f^{i-1}(S) \cap A$. Repeating this argument we find a point which is an $f^{i}$-preimage of $u$ and lies in $S$ and as before, the choice of $S$ implies that this point has at least one preimage outside $C$. This completes the proof of the claim.

Since $S$ is an $A$-covering unilateral neighborhood of $y^{\prime}$ then there are finitely many components of $\widetilde{S}$ such that the closure of their union contains $A$. This implies that $\widetilde{S}$ contains all of $A$ except for at most finitely many points. Moreover, $\widetilde{S} \backslash C \subset \widetilde{D}$ which implies that $\widetilde{D}$ contains all of $A \backslash C$ with the same possible exceptions. As $A$ contains infinitely many preimages of $u$ which are outside of $C$, $\widetilde{D}$ must contain at least one preimage of $u$. Hence, $u \in \widetilde{D}$ and it follows that $\widetilde{D}$ contains all of $A$ with the possible exception of finitely many points.

Now, the intervals $S, f(S), \ldots, f^{p}(S)$ are mapped onto $f^{p+1}(S)$ by the corresponding iterates of $f$. By the construction and Lemma 2.3 the orbit of each wandering component of $\widetilde{D}$ (if any) is disjoint from the intervals which precede it in $\mathbf{k}(D, U)$.

If one of the images of this wandering component intersects $C$ then a future image would intersect $f^{p+1}(S)$ and this is not the case; it follows that the wandering components of $\widetilde{D}$ and $\widetilde{S}$ are identical. Since wandering components of $\widetilde{S}$ do not contain points of $A$ we conclude that neither do the wandering components of $\widetilde{D}$. According to Lemma 2.3 there are finitely many non-wandering components of $\widetilde{D}$ and by what we saw in the previous paragraph their union contains all of $A$ with the possible exception of a finite set.

Let $x$ be such an exceptional point. If $x$ is not in the closure of the non-wandering components of $\widetilde{D}$ then $x$ is an isolated point of $A$. By Corollary $2.10 x$ is not periodic and has infinitely many preimages in $A$. But, some of these preimages must be contained in $\widetilde{D}$ and this implies that $x \in A$ contradicting the assumption. It follows that the closure of the non-wandering components of $\widetilde{D}$ contains $A$.

The fact that $D \subset R$ now implies that the set $\widetilde{R}$ has finitely many components such that the closure of their union contains $A$. Finally, since $R$ is an arbitrary $T$-unilateral neighborhood of $y, T$ is an $A$-covering side of $y$. Thus, $f^{p}\left(Z^{\prime}\right)$ always contains an $A$-covering unilateral neighborhood of $y$ which by Lemma 1.1 implies that there is a closed interval $V^{\prime} \subset V$ with the desired properties.

This completes the proof of the case when $n=2$. The general case follows by repeated use of this argument.

We're now in position to prove the main result of this section.

Theorem 2.12. A closed set $A$ is an $\omega$-limit set if and only if it is locally expanding.

Proof. We first show that if $A=\omega(x)$ is an $\omega$-limit set then $A$ is locally expanding. This is obvious if $A$ is a periodic orbit. If $A$ has non-empty interior then as $A$ is 
THE SPACE OF $\omega$-LIMIT SETS OF A CONTINUOUS MAP OF THE INTERVAL 1367

an $\omega$-limit set, $A$ is a cycle of intervals, i.e. $A=I \cup f(I) \cup \cdots \cup f^{n-1}(I)$ where $I$ is a periodic interval of period $n$. From this it follows that if $W \subset A$ is an interval, then $W$ has a dense orbit in $A$ and hence there is an $n \in \mathbb{N}$ such that $f^{n}(W) \cap f(W) \neq \emptyset$. Therefore the union $\bigcup_{i=1}^{\infty} f^{i}(W)$ is dense in $A$ and has but finitely many component intervals. As this is true for every such interval $W$, it follows that $A$ is locally expanding.

It remains to verify that if $A$ is a nowhere dense and infinite $\omega$-limit set, then $A$ is locally expanding. To do this, we prove that if $a \in A$ and $\operatorname{orb} x \mapsto a$ from the side, $T$, then $T$ is an $A$ covering side of $a$.

Let $U=U_{1} \cup \cdots \cup U_{n}$ be the union of closed pairwise disjoint intervals with $A \subset$ int $U$. Let $a \in A, T$ be a side such that $\operatorname{orb} x \mapsto a$ from $T$, and let $V \subset U$ be a closed $T$-semineighborhood of $a$. Clearly we may assume that $x \in V$ and orb $x \subset U$, and thus for any $n \in \mathbb{N}, f^{n}(x) \in U$ and $\operatorname{orb} x \subset \widetilde{V}$. By Lemma 2.3 the set $\widetilde{V}$ is the union of two disjoint sets:

$\mathcal{A}$, a finite family of disjoint intervals which are pieces of $\mathbf{k}(V, U)$ and

$\mathcal{B}$, a finite family of pairwise disjoint orbits of closed wandering intervals.

By our choice of $T$, the orbit of $x$ enters $V$ and therefore $f_{U}(V)$ infinitely many times. This implies that if $I$ is an interval whose orbit misses $\bigcup \mathcal{A}$, then orb $x \not \subset$ orbI.

According to Lemma 2.3 these orbits and $\mathcal{A}$ form the entire set $\widetilde{V}$ and by supplementing $\mathcal{A}$ (if necessary) by initial portions of wandering orbits we may assume that $\operatorname{orb} x \subset \cup \mathcal{A}$. Therefore $A=\omega(x) \subset \overline{\cup \mathcal{A}}$ and hence, $A$ is locally expanding. This completes the proof of the first half of the theorem.

Assume that $A$ is locally expanding. If $A$ is finite then by Corollary 2.10 it is a periodic orbit; if $A$ has a non-empty interior then by Lemma $2.6 A$ is a cycle of intervals and $f \mid A$ is transitive. These are well-known cases of $\omega$-limit sets, so from now on we assume that $A$ is nowhere dense and infinite. Our aim is to find a point $x$ such that $A=\omega(x)$. We will find this point by constructing a nested sequence of closed intervals whose orbits approximate the set $A$ more and more closely. Any point in the intersection of these intervals will have $A$ as its $\omega$-limit set.

Let $\left\{U^{n}: n \in \mathbb{N}\right\}$ be a sequence of finite unions of closed intervals such that

(1) $U^{1} \supset U^{2} \supset \ldots$,

(2) $U^{n}=U_{1}^{n} \cup U_{2}^{n} \cup \cdots \cup U_{k_{n}}^{n}$ where the $U_{i}^{n}$ are components of $U^{n}$ and intersect $A$,

(3) $A \subset$ int $U^{n}$ for each $n$,

(4) $\bigcap_{n=1}^{\infty} U^{n}=A$,

(5) $U_{1}^{n+1}$ is disjoint from $U_{k_{n}}^{n}$.

According to Proposition 2.11 there exists a point $z_{1} \in U_{1}^{1}$, a point $\zeta_{1} \in U_{k_{1}}^{1}$, a closed interval $V_{1} \subset U_{1}^{1}$ contained in an $A$-covering semineighborhood of $z_{1}$ and $\ell_{1} \in \mathbb{N}$ so that $\bigcup_{i=1}^{\ell_{1}} f^{i}\left(V_{1}\right) \subset U^{1}$, each component of $U^{1}$ contains at least one $f^{i}\left(V_{1}\right)$ and $W_{1}=f^{\ell_{1}}\left(V_{1}\right)$ is an $A$-covering semineighborhood of $\zeta_{1}$. Consider now the finite union of closed intervals which includes $U_{k_{1}}^{1}$ and all the components of $U^{2}$ which are disjoint from $U_{k_{1}}^{1}$ (in particular $U_{1}^{2}$ ). We apply Proposition 2.11 and Lemma 1.1 to find an interval $V_{1}^{\prime} \subset V_{1}$ and a number $r_{1}>\ell_{1}$ such that $\bigcup_{i=1}^{r_{1}} f^{i}\left(V_{1}^{\prime}\right) \subset U^{1}$ are contained in $U^{1}$, every component of $U^{1}$ contains at least one $f^{i}\left(V_{1}^{\prime}\right)$ and $W_{1}^{\prime}=f^{r_{1}}\left(V_{1}^{\prime}\right) \subset U_{1}^{2}$ is an $A$-covering semineighborhood of some point $z_{2} \in U_{1}^{2}$. Repeating this construction and applying Lemma 1.1 shows there is 
a sequence of closed intervals $V_{1}^{\prime} \supset V_{2}^{\prime} \supset \ldots$ and numbers $r_{0}=0<r_{1}<r_{2}<\ldots$ so that for each $n \in \mathbb{N}, \bigcup_{i=r_{n-1}}^{r_{n}-1} f^{i}\left(V_{n}^{\prime}\right) \subset U^{t}$ and every component of $U^{n}$ contains at least one $f^{i}\left(V_{n}^{\prime}\right)$.

The properties of the sequence of covers $\left\{U^{n}\right\}$ and the definition of the nested sequence $\left\{V_{n}^{\prime}\right\}$ now implies that if $x \in \bigcap_{n=1}^{\infty} V_{n}^{\prime}$, then $\omega(x)=A$. This completes the proof.

\section{Properties of the family of $\omega$-Limit sets Endowed With THE HAUSDORFF METRIC}

In Section 3 we obtain our main results which concern properties of the family of $\omega$-limit sets endowed with the Hausdorff metric.

Theorem 3.1. Let $\left\{\omega_{n}\right\}_{n=1}^{\infty}=\left\{\omega\left(x_{n}\right)\right\}_{n=1}^{\infty}$ be a sequence of $\omega$-limit sets of a continuous interval map $f$ and let a point a have a side $T$ such that for any $T$-unilateral neighborhood $V$ of a, there exists $N$ such that for $n \geq N$, the orbit of $x_{n}$ enters $V$ infinitely many times. ${ }^{1}$ Then $\bigcap_{k=1}^{\infty} \overline{\bigcup_{n=k}^{\infty} \omega_{n}}$ is an $\omega$-limit set.

Proof. Let $A_{k}=\overline{\bigcup_{n=k}^{\infty} \omega_{n}}$ and $A=\bigcap_{k=1}^{\infty} A_{k}$. It is easy to see that

$A=\left\{y:\right.$ in any neighborhood of $y$ there are points from infinitely many sets $\left.\omega_{n}\right\}$.

If $\omega_{n}=\omega_{n+1}$ for sufficiently large $n$ then the statement of the theorem is clear. So from now on we assume that $\omega_{n} \neq \omega_{n+1}$ for all $n$.

According to Theorem 2.12 it is enough to check that the set $A$ is locally expanding, that is, if $y \in A$, then $y$ has an $A$-covering side. Our method is to show that $T$ is an $A$-covering side of $a$ and then use this fact to find an $A$-covering side of other points of $A$. Let $U=U_{1} \cup \cdots \cup U_{n}$ be the union of closed intervals such that $A \subset$ int $U$, and let $V$ be a fixed closed $T$-unilateral neighborhood of $a$.

We first show that there are infinitely many $n$ for which the orbits of $x_{n}$ intersect int $V$ infinitely many times. If not, then the orbit of $x_{n}$ enters int $U$ only finitely many times for sufficiently large $n$ which implies that $x_{n}$ gets mapped into $a$ by some power of $f$. Therefore $\omega_{n}=\omega(a)$ for sufficiently large $n$ which contradicts the assumption that $\omega_{n} \neq \omega_{n+1}$.

Let us show that $T$ is an $A$-covering side of $a$. Let $N=N(V)$ be such that for $n \geq N$ the orbit of $x_{n}$ enters $V$ infinitely often. Let $z_{n}$ be a limit point of $\operatorname{orb} x_{n} \cap V$. Since both $\omega_{n}$ and $V$ are closed, $z_{n} \in \omega_{n} \cap V$. By Theorem 2.12 this implies that $V$ contains $z_{n}$ and its $\omega_{n}$-covering unilateral neighborhood, $R$. Working with the set $U$ and the corresponding map $f_{U}$ we use Theorem 2.12 to conclude that there are finitely many components of $\widetilde{V}$ such that whenever $n \geq N, \omega_{n}$ is in the closure of their union. Moreover, by Lemma 2.3 and Theorem 2.12 the rest of $\widetilde{V}$ consists of wandering closed intervals none of which intersects $\omega_{n}$. Therefore we may choose these components the same for all $n$ and we let $B$ be their union. It follows that $A_{n} \subset \bar{B}$ for $n \geq N$. Hence, $A \subset \bar{B}$ from which it follows that $T$ is an $A$-covering side of $a$. Note that from the definition it also follows that the orbit of $x_{n}$ is eventually contained in $B$.

Claim: Let $y \in A$, then there exists a side $S$ such that for any nondegenerate $S$-unilateral neighborhood $J$ of $y$ and for infinitely many integers $n$ the orbits of $x_{n}$ enter int $J$ infinitely many times.

\footnotetext{
${ }^{1}$ Note that a special case of this theorem is the case where there are points $a_{n} \in$ $\omega_{n}, a_{n} \neq a$ converging from the side $T$ to $a$.
} 
There are points from infinitely many sets $\omega_{n}$ in any fixed neighborhood $I$ of $y$, so there there is a sequence $\left\{n_{k}\right\} \subset \mathbb{N}$ such that for arbitrarily large powers $i, f^{i}\left(x_{n_{k}}\right) \in I$. If the side in question does not exist then we can choose $I$ sufficiently small that whenever $f^{i}\left(x_{n_{k}}\right) \in I, f^{i}\left(x_{n_{k}}\right)=y$. From this it follows that $\omega_{n_{k}}=\omega(y)$ for every $k \in \mathbb{N}$ which contradicts the assumption that $\omega_{n} \neq \omega_{n+1}$ for all $n$. This contradiction implies that the required side of $y$ exists, and we call this side a limit side of $y$.

If $S$ is such a limit side of $y$, then the fact that $B$ is the union of finitely many intervals and the orbit of every $x_{n}$ is eventually contained in $B$ implies that interiors of sufficiently small $S$-unilateral neighborhoods of $y$ are contained in $B$.

Claim: A limit side $S$ of $y \in A$ is an $A$-covering side of $y$.

As $S$ is a limit side of $y$, there is a sequence, $n_{k} \in \mathbb{N}$ with the property that for any open unilateral neighborhood $J$ of $y$ there exists an $N \in \mathbb{N}$ such that if $k>N$, the orbit of $x_{n_{k}}$ enters $J$ infinitely many times. Mirroring the formulation of $A_{k}$ and $A$, we define $D_{k}=\overline{\bigcup_{i=k}^{\infty} \omega\left(x_{n_{i}}\right)}$ and set $D=\bigcap_{k=1}^{\infty} D_{k}$. It follows from the definition that $a \in D$ and from what was proved above that the set $\widetilde{J}$ has finitely many components with union $G$ such that the closure of $G$ contains $D$ and the orbit of each $x_{n_{k}}$ is eventually contained in $G$. We show that $G$ contains the interior of a $T$-unilateral neighborhood of $a$. Indeed, otherwise orbits of all points $x_{n_{k}}$ which are eventually contained in $G$ cannot enter the interior of $V$ infinitely many times. Therefore all points $x_{n_{k}}$ are eventually mapped to $a$ which in this case is a periodic point. Now, shrink an $S$-unilateral neighborhood $J$ of $y$ so that the interior of the new $S$-unilateral neighborhood $J^{\prime}$ of $y$ contains no points from the periodic orbit of $a$. Then none of the points $x_{n_{k}}$ enters int $J^{\prime}$ infinitely many times and this is a contradiction.

Denote by $R$ a closed $T$-unilateral neighborhood of $a$ whose interior is covered by $G$. Since $T$ is an $A$-covering side of $a$ we conclude that there are finitely many components of $\widetilde{R}$ such that the closure of their union contains $A$. The fact that $R \subset G$ implies now that there are finitely many components of $\widetilde{J}$ whose union contains $A$ in its closure. As $y \in A$ was arbitrary, $A$ is locally expanding. This completes the proof of the theorem.

Remark 1. Theorem 3.1 generalizes the following two old results of Sharkovsky stated in [6].

(1) If $\omega_{1}, \omega_{2}$ are $\omega$-limit sets of $f$ with a common limit point from one side then their union is an $\omega$-limit set of $f$.

(2) If $\omega_{1} \subset \omega_{2} \subset \ldots$ is a sequence of $\omega$-limit sets of $f$ then the closure of their union is also an $\omega$-limit set of $f$.

Theorem 3.1 has a great deal to say about the relationship between an interval function and the corresponding space of $\omega$-limit sets endowed with the Hausdorff metric. We conclude this section with the theorem which was one of this study's main goals. 
Theorem 3.2. Let $f$ be a continuous interval map. Then the family of all $\omega$-limit sets of $f$ endowed with the Hausdorff metric is compact.

Proof. Let $\left\{\omega_{1}, \omega_{2}, \ldots\right\}$ be a sequence of $\omega$-limit sets converging in the Hausdorff metric to a set $A$. We may assume that $\omega_{n} \neq \omega_{n+1}$ for all $n$. Choosing a subsequence (if necessary) we may also assume that there exists a point $a$, a side $T$ of $a$ and points $a_{n} \in \omega_{n}, a_{n} \neq a$ converging to $a$ from $T$. As the original sequence converged to $A$, the subsequence does as well. The fact that $A$ is an $\omega$-limit set now follows from Theorem 3.1.

\section{Some Applications}

A main question of the paper was whether $\omega$-limit sets of an interval map form a closed family in the Hausdorff metric. Clearly this question can be asked for other dynamical systems too. It turns out that in some cases one can deduce the answer from Theorem 3.2.

Let us consider the following class of dynamical systems. Set $I=[0,1]$ and suppose $f: I \rightarrow I$ is continuous. Let $U_{1}=\left[a_{1}, b_{1}\right], U_{2}=\left[a_{2}, b_{2}\right], \ldots, U_{k}=\left[a_{k}, b_{k}\right]$ be a finite family of closed pairwise disjoint intervals, $U=\bigcup_{i=1}^{k} U_{i}$, and consider the set

$$
A_{U}(f)=\left\{x: \operatorname{orb}_{f}(x) \subset U\right\} .
$$

It is easy to see that the set $A_{U}(f)$ is closed and invariant. This particular set is sometimes studied under the assumption that $f$ is piecewise-monotone, but we require no conditions other than continuity.

Theorem 4.1. The family of $\omega$-limit sets of $f \mid A_{U}(f)$ is closed in the Hausdorff metric.

Proof. As a first case, we consider the situation where one of the component intervals of $U$ is degenerate, say $U_{i_{o}}=\{a\}$ (that is, $a_{i_{o}}=b_{i_{o}}=a$ ). Let

$$
U^{\prime}=\bigcup_{\substack{i=1 \\ i \neq i_{o}}}^{k} U_{i}
$$

CASE 1. $a \notin A_{U}(f)$

In this case, the families of $\omega$-limit sets of $f \mid A_{U^{\prime}}(f)$ and $f \mid A_{U}$ are identical, so we can simply replace $U$ by $U^{\prime}$.

CAse 2. $a \in A_{U}(f)$

If $a$ is not periodic then $\omega_{f}(a)=\omega_{f}(f(a))$ which again implies that the family of $\omega$-limit sets of $f \mid A_{U}(f)$ is the same as that of $f \mid A_{U^{\prime}}(f)$. If $a$ is periodic then the family of all $\omega$-limit sets of $f \mid A_{U}(f)$ is that of $f \mid A_{U^{\prime}}(f)$ united with $\operatorname{orb}_{f}(a)$. However, the $\operatorname{orb}_{f}(a)$ is an isolated point in the family of all $\omega$-limit sets of $f \mid A_{U}(f)$ since the distance between $\operatorname{orb}_{f}(a)$ and another $\omega$-limit set of $f \mid A_{U}(f)$ is greater than the minimal distance between $a$ and any other point from $U$.

Hence, it suffices to consider the case where no $U_{i}$ is degenerate.

Thus we may assume that $a_{1}<b_{1}<a_{2}<b_{2}<\cdots<a_{k}<b_{k}$. Our method is to define a relatively tame function $g: I \rightarrow I$ which agrees with $f$ on $A_{U}$ and then to apply Theorem 3.1. For convenience, define $b_{0}=0$ and $a_{k+1}=1$. Let 
THE SPACE OF $\omega$-LIMIT SETS OF A CONTINUOUS MAP OF THE INTERVAL 1371

$\varepsilon=\min \left\{a_{i+1}-b_{i}: i=0,1, \ldots k a_{i+1}-b_{i}>0\right\} / 3$ and define

$$
g(x)= \begin{cases}f(x), & x \in U \\ f\left(a_{i}\right), & a_{i}-\varepsilon \leq x \leq a_{i} \\ f\left(b_{i}\right), & b_{i} \leq x \leq b_{i}+\varepsilon \\ \text { linear } & \text { otherwise }\end{cases}
$$

Then, $g: I \rightarrow I$ is continuous map and it's easy to see that $A_{U}(f)=A_{U}(g)=A$ and $f|A=g| A$. Let $W=U \cup \bigcup_{i=1}^{k}\left(a_{i}-\epsilon, a_{i}\right] \cup\left[b_{i}, b_{i}+\epsilon\right)$.

To prove that $f \mid A_{U}$ has the property that all its $\omega$-limit sets form a closed family in the Hausdorff metric one has to show that if $\omega_{f}\left(x_{n}\right), n \geq 1$, is the sequence of $\omega$ limit sets of points $x_{n} \in A$ such that for some set $R$ we have $H\left(\omega_{f}\left(x_{n}\right), R\right) \rightarrow 0$ then there exists a point $x \in A$ such that $R=\omega_{f}(x)$. As $f|A=g| A, \omega_{f}\left(x_{n}\right)=\omega_{g}\left(x_{n}\right)$ and $f\left|\omega_{f}\left(x_{n}\right)=g\right| \omega_{f}\left(x_{n}\right)$. Hence, $H\left(\omega_{g}\left(x_{n}\right), R\right) \rightarrow 0$ and by Theorem 3.1 there is an $x \in[0,1]$ such that $\omega_{g}(x)=R$. It remains to show that this $x$ can be taken in $A$ and for this it is sufficient to find an $m$ such that $g^{m}(x) \in A$. As $R=\omega_{g}(x) \subset A$, there exists $N$ such that for $n \geq N, g^{n}(x) \in W$. If our assumption that $g^{m}(x) \in A$ for some $m$ fails then there exist infinitely many integers $k$ such that $g^{k}(x) \notin U$. The construction of the map $g$ implies that in this case for some $k$ we have that $g^{k}(x)$ is a $g$-periodic point and on the other hand $g^{k}(x) \notin U$. Therefore $\omega_{g}(x) \notin U$ contradicting the fact that $\omega_{g}(x)=R \subset U$. Since by the construction $f|A=g| A$ we conclude that $R=\omega_{f}(x)$ which completes the proof.

It is easy to see now that one-sided subshifts of finite type have the property that their family of $\omega$-limit sets is closed. Indeed, the following corollary holds.

Corollary 4.2. If $\sigma: S \rightarrow S$ is a one-sided subshift of finite type then the family of all its $\omega$-limit sets is closed in the Hausdorff metric.

Proof. Let $S$ be defined by a $0-1$-matrix $\left(s_{i j}\right), 1 \leq i \leq n, 1 \leq j \leq n$, so that the sequence $\mathbf{v}=\left(v_{0}, v_{1}, \ldots\right), 1 \leq v_{i} \leq n$, belongs to $S$ if and only if $s_{v_{i} v_{i+1}}=1$. Consider a continuous map $f:[0,1] \rightarrow[0,1]$ defined as follows:

$$
f(x)= \begin{cases}0, & x=i / n \text { for } 0 \leq i \leq n \\ 1, & x=(2 i+1) /(2 n) \text { for } 0 \leq i \leq n-1 \\ \text { linear } & \text { on }[j / 2 n,(j+1) / 2 n] \text { for } 0 \leq j \leq 2 n-1\end{cases}
$$

Let $J_{i}=[i / n,(2 i+1) / 2 n]$, and for any $1 \leq i \leq n, 1 \leq k \leq n$, with $s_{i k}=1$ let $M_{i k} \subset J_{i}$ be a closed interval such that $f\left(M_{i k}\right)=J_{k}$. Then, $M_{i k}$ is well-defined and two different intervals of this type are disjoint.

Let $U$ denote the union of all intervals $M_{i k}$. Then, every point $z \in A_{U}(f)$ generates a sequence $\mathbf{v}=\left(v_{0}, \ldots\right)=\phi(z)$ so that $f^{r}(z) \in J_{v_{r}}$ for any $r \geq 0$. That is, $f \mid A_{U}(f)$ and $\sigma \mid S$ are topologically conjugate. No two points are mapped into one sequence because of the expanding properties of $f$. On the other hand, for any sequence $\mathbf{v} \in S$ there exists a point $z \in A_{U}(f)$ such that $\phi(z)=\mathbf{v}$. Indeed, let $Z_{m}=\bigcap_{k=0}^{m} \phi^{-k}\left(M_{v_{k} v_{k+1}}\right)$ and $Z=\bigcap_{k=0}^{\infty} Z_{m}$. As the $Z_{m}$ are nested, $Z$ is nonempty and therefore, $\phi$ is a homeomorphism between $A_{U}(f)$ and $S$ which conjugates $f \mid A_{U}(f)$ and $\sigma \mid S$. It follows that the family of all $\omega$-limit sets of $\sigma \mid S$ is closed in the Hausdorff metric.

Lemma 4.3. Let $X$ and $Y$ be compact metric spaces, $f: X \rightarrow X, g: Y \rightarrow Y$ be continuous, and suppose that $\phi: X \rightarrow Y$ is a surjective semiconjugacy between $f$ 
and $g$. If the set of all $\omega$-limit sets of $f$ is closed in the Hausdorff metric, then so is the set of all $\omega$-limit sets of $g$.

Proof. Suppose that $y_{n} \in Y$ is a sequence of points such that the sequence of their $\omega$-limit sets $\omega_{g}\left(y_{n}\right)$ converges in the Hausdorff metric to a set $A$. We have to show that $A=\omega(y)$ for some $y$. To this end let $y_{n}=\phi\left(x_{n}\right)$; clearly $\omega_{g}\left(y_{n}\right)=\phi\left(\omega_{f}\left(x_{n}\right)\right)$ for any $n$. Choosing a subsequence we may assume that $\omega_{f}\left(x_{n}\right)$ is a sequence which converges in the Hausdorff metric to a set $B$ and by the assumption about $f$ there exists a point $x$ such that $\omega_{f}(x)=B$. It is obvious that $A=\phi(B)=\phi\left(\omega_{f}(x)\right)=$ $\omega_{g}(\phi(x))$ so if we set $y=\phi(x)$ then we will have $A=\omega_{g}(y)$ which completes the proof.

Suppose that a dynamical system is a factor of a one-sided subshift of finite type (a sofic system is an example of such a factor) or more generally of a map $f \mid A_{U}$ where $U$ is the union of a few pairwise disjoint closed intervals and $f$ is a continuous interval map. Then the results of this section apply to this dynamical system. This shows that in fact the family of all $\omega$-limit sets is closed in the Hausdorff metric for a large variety of dynamical systems.

\section{REFERENCES}

1. S. J. Agronsky, A. M. Bruckner, J. G. Ceder and T. L. Pearson, The structure of $\omega$-limit sets for continuous functions, Real Analysis Exchange 15 (1989-1990), 483-510. MR 91i:26008

2. L. S. Block and W. A. Coppel, Dynamics in One Dimension, Lecture Notes in Mathematics, vol. 1513, Springer, Berlin, 1992. MR 93g:58019

3. A. M. Bruckner and J. G. Ceder, Chaos in terms of the map $x \rightarrow \omega(f, x)$, Pac. J. Math. 156 no. 1 (1992), 63-96. MR 93g:58092

4. A. M. Bruckner and J. Smital, The structure of $\omega$-limit sets for continuous maps of the interval, Mathematica Bohemica 117 (1992), 42-47. MR 93a:26002

5. A. N. Sharkovsky, Continuous maps on the set of limit points of an iterated sequence, Ukr. Math. J. 18 no. 1 (1966), 127-130. MR 34:6732

6. _ The partially ordered system of attracting sets, Soviet Math. Dokl. 7 no. 5 (1966), 1384-1386. MR 35:311

Department of Mathematics, University of Alabama in Birmingham, University Station, Birmingham, Alabama 35294-2060A

E-mail address: ablokh@math.uab.edu

Department of Mathematics, University of California at Santa Barbara, Santa BarBara, CALIFornia 93106

E-mail address: bruckner@math.ucsb.edu

Department of Mathematics, St. Olaf College, Northfield, Minnesota 55057

E-mail address: humke@stolaf.edu

Institute of Mathematics, Silesian University, 74601 Opava, Czech Republic

E-mail address: smitalum@fpf.slu.cz 